\title{
Relating Ventilatory Support and Drug Treatment Strategies to the Fundamental Pathophysiology in COVID-19 IIIness
}

\author{
Authors: \\ * Keir E. Lewis, ${ }^{1,2}$ Alexander Stoll, ${ }^{3}$ Sarah Watts, ${ }^{3}$ Emrys Kirkman ${ }^{3}$ \\ 1. Department of Medicine, Prince Philip Hospital, Llanelli, UK \\ 2. College of Human and Health Sciences, University of Swansea, Swansea, UK \\ 3. CBR Division, Defence Science and Technology Laboratory, Salisbury, UK \\ *Correspondence to Keir.lewis@wales.nhs.uk \\ Disclosure: $\quad$ The authors have declared no conflicts of interest. \\ Acknowledgements: The authors are grateful to Dr Jess Skelton for drawing Figure 1 and 2, and the \\ staff and patients on the COVID-19 wards at Prince Philip Hospital, Llanelli, UK, for \\ recording the clinical data for Figure 3. \\ Received: \\ 21.11.20 \\ Accepted: \\ 02.02 .21 \\ Keywords: \\ Alveolar inflammation, continuous positive airway pressure (CPAP), coronavirus \\ disease (COVID-19), drug treatments, endothelial dysfunction, pneumonitis. \\ Citation: \\ EMJ. 2021; DOI/10.33590/emj/20-00269
}

\begin{abstract}
This article relates the current pathophysiologic and radiologic findings to the fundamental idea of acute lung epithelial infection, alveolar inflammation causing leak into the interstitial space, and subsequent secondary or concurrent endothelial infection and dysfunction. Understanding the mechanisms and timings of alveolar damage can better inform the types of ventilatory support required and timing of targeted pharmacotherapies.
\end{abstract}

\section{INTRODUCTION}

Coronavirus disease (COVID-19) is caused by severe acute respiratory syndrome coronavirus 2 (SARS-CoV-2); ${ }^{1}$ as of September 2020, there were $>33$ million cases and almost 1 million deaths worldwide. ${ }^{2}$ An unknown number of people are asymptomatic carriers of SARS-CoV-2 but symptoms in the early phase of viral replication include fatigue, anosmia, fever, sore throat, and cough. Around $85-95 \%$ of people make an uncomplicated recovery within 10 days; however, some individuals develop worsening cough, dyspnoea, and persistent (higher) fever, some of whom require hospital admission with hypoxia and interstitial pneumonia. ${ }^{3}$ This more severe illness is from viral infection within the lung tissue and the associated immune dysfunction, with loss of regulation of the inflammatory cascade leading to further lung damage, worsening hypoxia, and other organ dysfunction.

\section{FUNDAMENTAL PATHOPHYSIOLOGY}

Two possible inflammation mechanisms (not mutually exclusive) can result in fluid accumulation in the lung alveoli: 1) direct viral and immune-mediated damage to the epithelium, affecting alveolar movement and reducing pulmonary fluid clearance; 2) a secondary or 
parallel injury to the endothelium or its basement membrane that results in capillaritis with further leak of fluid from the plasma into the interstitial space and thereon into the air spaces.

\section{IMPACT OF EPITHELIAL DAMAGE}

SARS-CoV-2 targets and damages pulmonary epithelium, ${ }^{4}$ particularly ciliated airway epithelial cells and Type 2 pneumocytes through the abundant angiotensin-converting enzyme 2 receptors in the lung. The virus relies on cell membrane serine protease TMPRSS2 cleaving and subsequently activating S-proteins and furine protease activity. ${ }^{5}$ Hydrostatic and osmotic forces determine fluid movement from the vascular space to the interstitium through the extracellular matrix and the endothelial glycocalyx layer. Once the normally tight alveolar epithelial barrier is breached, alveolar oedema ensues. The epithelium also performs an important function in reabsorption of fluid through the asymmetric distribution of sodium channels and pumps throughout the alveoli and small airways. Sodium from the alveolar fluid passively enters the apical part of epithelial cells via the sodium channel and is pumped out of the cell via a sodium-potassium pump on the basal membrane. This shift of sodium causes an osmotic gradient leading to movement of water out of the alveoli, avoiding or resolving oedema in the alveolar air space (Figure 1). ${ }^{6}$ Inflammatory damage that increases permeability of the epithelial barrier or a reduction in the number of epithelial cells, channels, and/ or pumps would therefore impair an important clearance mechanism that helps avoid or resolve pulmonary oedema. Hypoxia itself is extremely proinflammatory and can further exacerbate tissue damage through cytokine release in a selfperpetuating and rapidly escalating autoimmune cycle of harm. Hypoxia further attenuates the basal pumps' highly energy-dependent sodiumpotassium pump clearance mechanism.

\section{POTENTIAL VENTILATION OR VENTILATOR DAMAGE TO THE PULMONARY ENDOTHELIUM OR EPITHELIUM}

Normal breathing at tidal volume can further stretch damaged and friable epithelium or endothelium and increase the permeability further. Excessive movements of damaged alveolar units through mechanical stress or sheer stress exacerbate extravasation of fluid, worsening hypoxia. In this regard, the authors propose that ventilatory support strategies should allow enough alveolar capillary oxygenation with a minimal degree of alveolar movement. Reducing physical movement of a leaky epithelium would reduce leak, attenuate further hypoxia, and buy more time for the body to clear the virus or for the antivirals to take effect, and for timely, targeted immunosuppression to reduce bystander cellular damage.

\section{EVIDENCE FROM PULMONARY IMAGING}

CT chest scans report ground-glass opacities, often together with areas of consolidation and lung interlobular septal thickening, predominantly in subpleural and middle/lower lung fields. ${ }^{7-10}$ Plain chest radiographs show multiple patchy shadows, $7,8,11$ and ultrasounds report B-lines ranging "across a continuum from mild alveolar interstitial pattern, severe bilateral infiltration pattern to lung consolidation". ${ }^{2}$

Serial CT scans show early subpleural bilateral patchy focal infiltrates, which coalesce into large confluent patchy areas, becoming geographically connected in a 'crazy-paving' pattern and ultimately progressing to diffuse lung consolidation. ${ }^{13,14}$ These sequential images represent progressive areas of alveolar epithelial damage, consistent with a spreading infection and local associated immune reaction. This patchy distribution supports an aerogenous route of pulmonary infection. ${ }^{15}$ CT scans usually correlate with clinical severity, ${ }^{7}$ but unlike traditional models of acute respiratory distress syndrome (ARDS) there can be severe radiological changes in relatively well and even asymptomatic people. Conversely, some symptomatic patients may present with a normal CT, especially in the early phase. ${ }^{16}$

Imaging in acute COVID-19 is entirely consistent with patchy progressive infection and distal alveolar inflammatory leak. ${ }^{12}$ Pleural effusions are rare in COVID-19 because the leak into the third space is not due to hydrostatic back-pressure, but due to small multifocal areas of patchy alveolar epithelial and endothelial damage. ${ }^{7,9}$ 


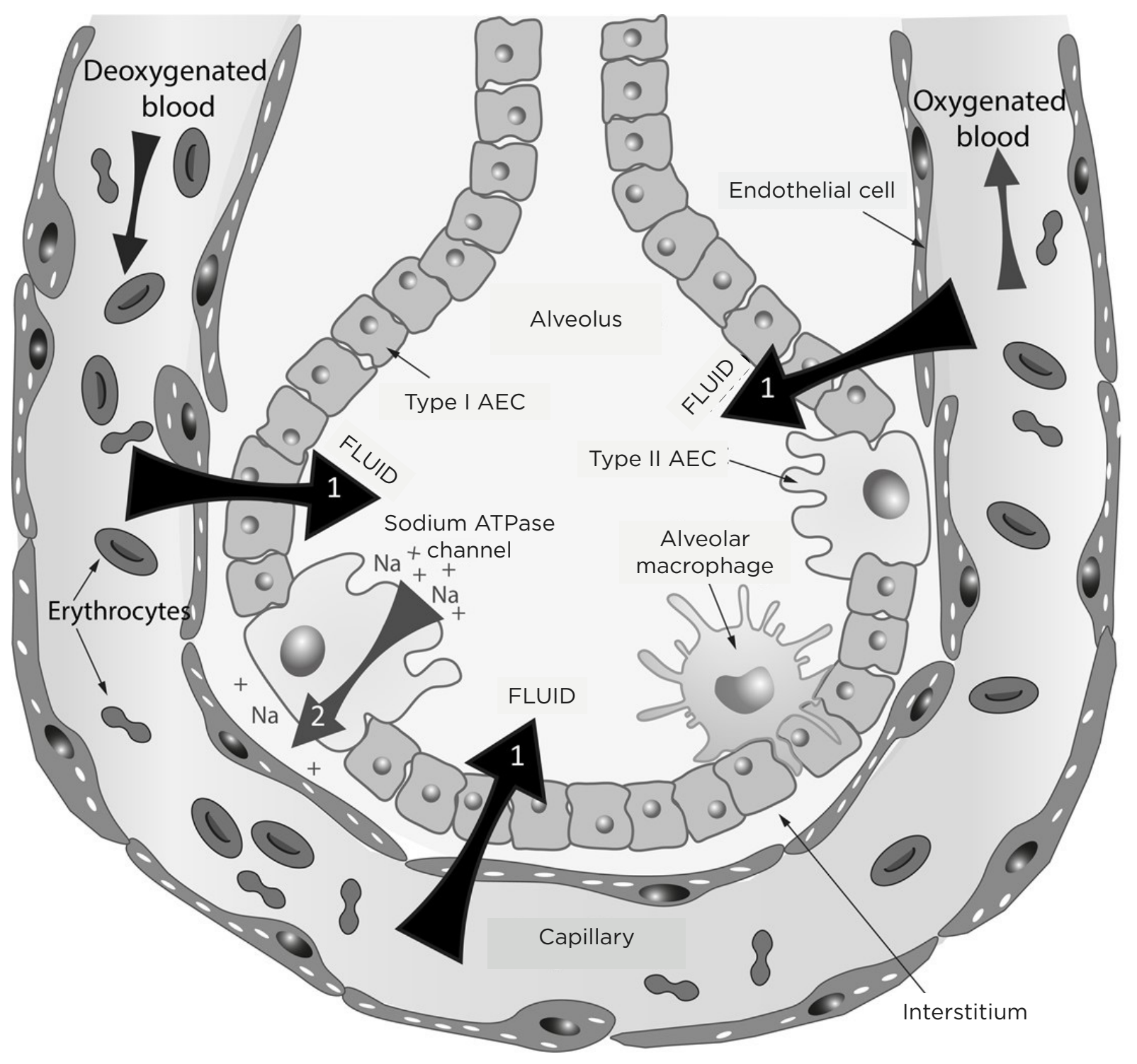

Figure 1: Fluid movements across the alveolar-capillary barrier (black and grey arrows).

AEC: alveolar epithelial cells; Na: sodium.

\section{EVIDENCE FROM GROSS LUNG PATHOLOGY}

Gross thoracic changes include uneven mottled red to blue-red colour, increased lung weight, oedema, severe congestion, pleuritis, consolidation thrombi, and often secondary bacterial infection. ${ }^{17,18}$

\section{EVIDENCE FROM LUNG HISTOPATHOLOGY}

Antemortem histopathological changes were seen coincidentally in samples of lung tissue taken for other reasons. Findings included alveolar oedema, fibrinous exudate, inspissated protein, haemorrhage, mononuclear inflammation, and multinucleated giant cell formation. ${ }^{18,19}$ Two patients developed severe COVID-19, and one of these patients died. ${ }^{20}$

Postmortem histopathological changes included desquamation of the alveolar epithelium, reactive Type 2 pneumocyte hyperplasia, intra-alveolar exudates with organising fibrin, hyaline membrane formation, haemorrhage, excess lymphocytes, neutrophils, alveolar megakaryocytes, thrombosis of small and midsized pulmonary arteries, and some interstitial fibrosis..$^{14,21-23}$ From the autopsies of 21 individuals 
who died as a result of COVID-19, the primary cause of death was reported as respiratory failure, with exudative diffuse alveolar damage, and with severe capillary congestion and microthrombi in alveolar capillaries. Pulmonary embolism $(n=4)$, alveolar haemorrhage $(n=3)$, vasculitis $(n=1)$, and superimposed bronchopneumonia $(n=10)$ were also reported. ${ }^{18}$

The inflammation is predominantly lymphocytic with large atypical pneumocytes," probably caused by viral cytopathic changes.11,18,24 Macrophages and monocytes are increased in alveolar spaces, and virus particles or antigens are detected in the epithelial cells, Type 1 and 2 pneumocytes, and macrophages by electron microscopy, immunohistochemistry, and in situ hybridisation. ${ }^{21}$

The primary damage occurs mainly to the alveolar epithelial cells. Initially, some research proposed that the lack of direct damage to endothelial cells may partly explain why other organs (heart: 7-9\%; kidney: 3-15\%) are less affected than the lungs. ${ }^{25-28}$ However, COVID-19 clearly incorporates other organ dysfunction and much more so than in other coronaviruses (severe acute respiratory syndrome and Middle East respiratory syndrome) and influenza. ${ }^{29}$

Endothelial cell viral infection, inflammatory cell infiltration, apoptosis of endothelial and mononuclear cells with widespread thrombosis, ${ }^{30}$ microangiopathy, marked increase in fibrinous microthrombi, and both intussusceptive and conventional sprouting angiogenesis ${ }^{31}$ have been reported. In one systematic pulmonary dissection study, all patients exhibited thrombosis of small or midsized pulmonary arteries. ${ }^{22}$ Here, the authors postulate that direct infection of endothelial cells does occur and their close proximity to the epithelium in the lung is why the lung is affected most by local vascular dysregulation, 22,30,32 leading to further hypoxia without loss of lung compliance in the early phase.

\section{EVIDENCE FROM PATHOPHYSIOLOGY}

\section{Intubated Patients}

Standard ARDS Berlin criteria require an onset within 7 days of a clinical insult or respiratory symptoms; ${ }^{33}$ however, in COVID-19, respiratory failure occurs typically 10-15 days after onset of symptoms. ${ }^{25-28}$ Early cohorts had worse outcomes with over-inflation ${ }^{34,35}$ and higher levels of barotrauma compared to ventilated patients with standard ARDS. ${ }^{35}$ Current COVID-19 ventilator guidelines consistently recommend lower pressures, probably to reduce alveolar stretch. ${ }^{36,37}$

Unlike most people with ARDS, many patients with COVID-19 receiving ventilation (especially early on) have compliant lungs despite profound oxygenation failure. 38,39 Some studies have defined separate phenotypes in COVID-19 pneumonitis according to lung compliance, ${ }^{40}$ but whether these are truly distinct or sequential stages of the same disease is in debate. Relatively high compliance is associated with high shunt fractions (50\%), rarely seen in other forms of ARDS. ${ }^{40}$ Again, this supports the idea that disrupted vasoregulation is an important contributor to poor oxygenation, at least in the early stages of COVID-19 lung injury. A major problem is the failure of the normal hypoxiainduced pulmonary vasoconstriction, ${ }^{41}$ which depends on good endothelial function, leading to hyperperfusion of 'gasless tissue'. CT scans report areas of dilated vessels in parts of the lung that are unlikely to be well ventilated. ${ }^{10,41}$ The improvement of oxygenation with positive endexpiratory pressure and prone positioning may be due to haemodynamic adjustment through local fluid shifts and better alveolar recruitment. ${ }^{39}$

The origin of the 'preserved' compliance is unknown; while it is probably beneficial for ventilation, it may have other detrimental effects. The lung possesses a highly efficient mechanism to minimise interstitial fluid volume. The pulmonary capillary basement membrane normally has low permeability to water due to the macromolecular organisation of heparan sulfate proteoglycans. Furthermore, any increase in extravascular fluid is rapidly cleared because of the high elastance (poor compliance) of the extracellular matrix consisting of a second group of matrix chondroitin sulfate proteoglycans. Hypoxia causes fragmentation of both sets of proteoglycans, resulting in decreased elastance (increased compliance) and leading to more alveolar movement and potential oedema. ${ }^{42}$ Applying high levels of positive end-expiratory pressure may accentuate underlying alveolar and microvascular injury and contribute to a worse outcome. ${ }^{43}$ 
The authors suggest that the respiratory distress seen in COVID-19 starts with a primary epithelial failure, followed by a secondary or concurrent endothelial vascular dysfunction. Therefore, ventilation should be guided by daily changes in radiology and estimates of capillary wedge pressure and lung water. Cardiac dysfunction from viral-induced myocarditis and/or hypoxaemia, worsening any pre-existing cardiac conditions, may also complicate vascular filling. Various fluid balance strategies have been tried in typical ARDS, based on estimated pulmonary capillary wedge pressure and extravascular lung water, but the unusual pulmonary haemodynamics seen in COVID-19 are a further complicating factor in the cardiac-pulmonary-specific interactions in intubated patients.

The wide variations in mortality of intubated patients with COVID-1926,38,39 could be due to fixed ventilatory strategies and basing pressure or target volumes on traditional ARDS models. The authors argue that the traditional (Berlin) definitions of ARDS do not align with COVID-19 respiratory failure in terms of diagnosis or, more importantly, ventilator management of lower pressures and daily changes according to variable haemodynamics and hypoxaemia. Extracorporeal membrane oxygenation (ECMO) is increasingly used for adult patients with potentially reversible respiratory failure refractory to conventional management. ECMO beds have been increased worldwide in response to the pandemic and some countries have issued guidelines to assist in identifying eligible patients and management. ${ }^{44}$ However, most clinicians in the ECMO service agree that the current evidence base does not allow strict criteria to determine optimal benefit and therefore encourage early referral. In reality, the experience (of the authors) has been that decisions are often made according to ECMO availability.

\section{Awake Patients}

Hypoxic patients with COVID-19 are commonly seen taking rapid and shallow breaths. ${ }^{45}$ Increased pulmonary interstitial pressure or volume activates pulmonary afferent C-fibre receptors, which are known to initiate a reflex that includes rapid shallow breathing and dyspnoea. ${ }^{46}$ These receptors are stimulated by increases in pulmonary interstitial pressure or volume (as occurs in pulmonary oedema). The combination of hypoxia, hypocapnia, tachypnoea and pulmonary oedema as seen in altitude sickness, ${ }^{47}$ traumatic 'blast lung', 48 and chemical lung injury ${ }^{49}$ are explained by the increased minute ventilation in response to the hypoxia; there is a disproportionate elimination of $\mathrm{CO}_{2}$ because its exchange is not impaired by pulmonary oedema as much as oxygen.

Awake patients with COVID-19 tolerate extreme hypoxia surprisingly well. Many patients converse freely despite peripheral oxygen saturations of $80-92 \%$ but have respiratory rates of $30-$ 38 breaths per minute, suggesting that rapid, shallow breathing is a natural response. Deeper inspiration causes dyspnoea and often back or chest pain in individuals with radiological COVID-19 pneumonitis (personal observation from Lewis KE).

However, if patients experience worsening hypoxia and start taking strong, spontaneous inspiratory efforts, this can physically stretch an already damaged alveolar epithelium and increase tissue stresses (akin to patient selfinflicted lung injury). ${ }^{50}$ Coexistent endothelial dysfunction with ventilation/perfusion mismatch leads to rapidly worsening hypoxaemia, more inflammation, more leak, and more hypoxia.

However, one notable difference is that dyspnoea is much less pronounced in COVID-19. Fifteen percent of patients with mild-case COVID-19 had shortness of breath, and only $38 \%$ of severe cases in China $^{27}$ and $17 \%$ of hospitalised cases in New York, USA, with tachypnoea ${ }^{51}$ complained of breathlessness. Whether this is of any significance in terms of respiratory control mechanisms remains to be seen.

Patients experiencing mild COVID-19 pneumonitis often have rapid, shallow breathing at rest, with normal arterial oxygen saturation. Frequently in these patients, mild exercise precipitates peripheral oxygen desaturation, consistent with an initial small reduction in pulmonary capillary gas transfer, which is sufficient to activate the pulmonary afferent C-fibre receptors and alter the breathing pattern. ${ }^{46}$ In normal lungs at rest, however, because the blood becomes fully saturated with oxygen by the time it has travelled approximately one-third the length of the pulmonary capillary, there is sufficient reserve to allow nearly complete saturation 
when the patient is at rest. ${ }^{52}$ However, with mild exercise the increased cardiac output reduces pulmonary blood transit time, so that pulmonary gas transfer now becomes diffusion-limited with consequent arterial desaturation. During recovery and rehabilitation, patients still profoundly desaturate, suggesting that it takes some time for the pulmonary capillary diffusion barriers to completely resolve.

\section{SUPPORTING EVIDENCE FROM CONTINUOUS POSITIVE AIRWAY PRESSURE}

Despite early experience with invasive ventilation, many healthcare professionals are moving towards noninvasive ventilation strategies for hypoxic COVID-19.53 Mild-to-moderate oxygenation failure in COVID-19 responds to high-flow nasal oxygen (HFNO) systems or continuous positive airway pressure (CPAP). ${ }^{3}$ These treatments allow the patient to eat, drink, communicate, and self-position. By reducing the work of breathing and inspiratory effort, it can reduce mechanical sheer stress, ventilationinduced lung injury, tracheostomy, ventilationassociated pneumonia, and difficulty in weaning. CPAP may delay or avoid the need for mechanical ventilation in patients with COVID-19. ${ }^{27,53-55}$ When intensive treatment unit resources are scarce, inappropriate use of invasive ventilation may stop access to life-saving treatment to those that need it. CPAP is part of the UK recommendations for the management of COVID-19.41,56 It is effective in the treatment of chemical lung injury with pulmonary oedema, reduced gas transfer, and high shunt fraction. ${ }^{57}$

These pathophysiological mechanisms explain how a ventilatory support strategy using CPAP and HFNO maintains both a physical pressure gradient and a partial-pressure gradient to allow continuous oxygen diffusion down the airways and across gently splinted-open alveoli that do not need to move much. This also allows rapid, small breaths to minimise movement of damaged epithelial and endothelial interfaces and supports fatigued muscles. Combining CPAP with HFNO allows maximum diffusion at the alveolar-capillary interface with minimum alveolar movement (Figure 2).

\section{ADDITIONAL IMPORTANT TREATMENTS}

Adequate fluid replacement is important as patients are prone to dehydration through increased insensible losses (fever, tachypnoea, occasionally diarrhoea) and reduced oral intake (nausea, CPAP masks).

Prone positioning can help oxygenation in some patients (while awake, on CPAP and ventilators). Presumably, prone positioning helps recruit more or different alveoli and therefore aligns with the hypothesis of localised inflammation and alveolar leak, rather than alveolar oedema from heart failure or increased hydrostatic pressure, which often worsen when lying supine or prone.

Management of coexistent medical conditions, especially diabetes, cardiac dysfunction, pulmonary emboli, and superadded bacterial or fungal lung infections, is also crucial. Worsening symptoms (including new confusion), worsening hypoxia, higher ventilatory pressure support, new raised temperature, rising inflammatory markers (neutrophils and C-reactive protein), raised procalcitonin, and especially focal consolidation and fluid or cavities on chest X-rays or CT scans, should alert clinicians to coincidental/secondary infections. Bacterial and fungal infections are reported in at least $10-30 \%$ of postmortem lung specimens of people who died of COVID-19.18,21-23

\section{SUPPORTING EVIDENCE FROM DRUG TREATMENTS}

A detailed review of all current pharmacological treatments is beyond the scope of this article. However, the predominant success so far of immunosuppressant and immunomodulatory drugs fits with the idea of reducing primary inflammatory epithelial/endothelial damage. The UK multicentre RECOVERY trial showed dexamethasone reduced deaths in ventilated patients (rate ratio: 0.65; 95\% confidence interval $[\mathrm{Cl}]$ : 0.48-0.88; $\mathrm{p}=0.0003$ ) and deaths in patients receiving oxygen (rate ratio: 0.80; 95\% Cl: 0.67$0.96 ; p=0.0021$ ). There was no benefit among those patients not requiring respiratory support (rate ratio: $1.22 ; 95 \% \mathrm{Cl}: 0.86-1.75 ; \mathrm{p}=0.14)^{58}$ presumably because there was no bystander immune-related damage at this point. 


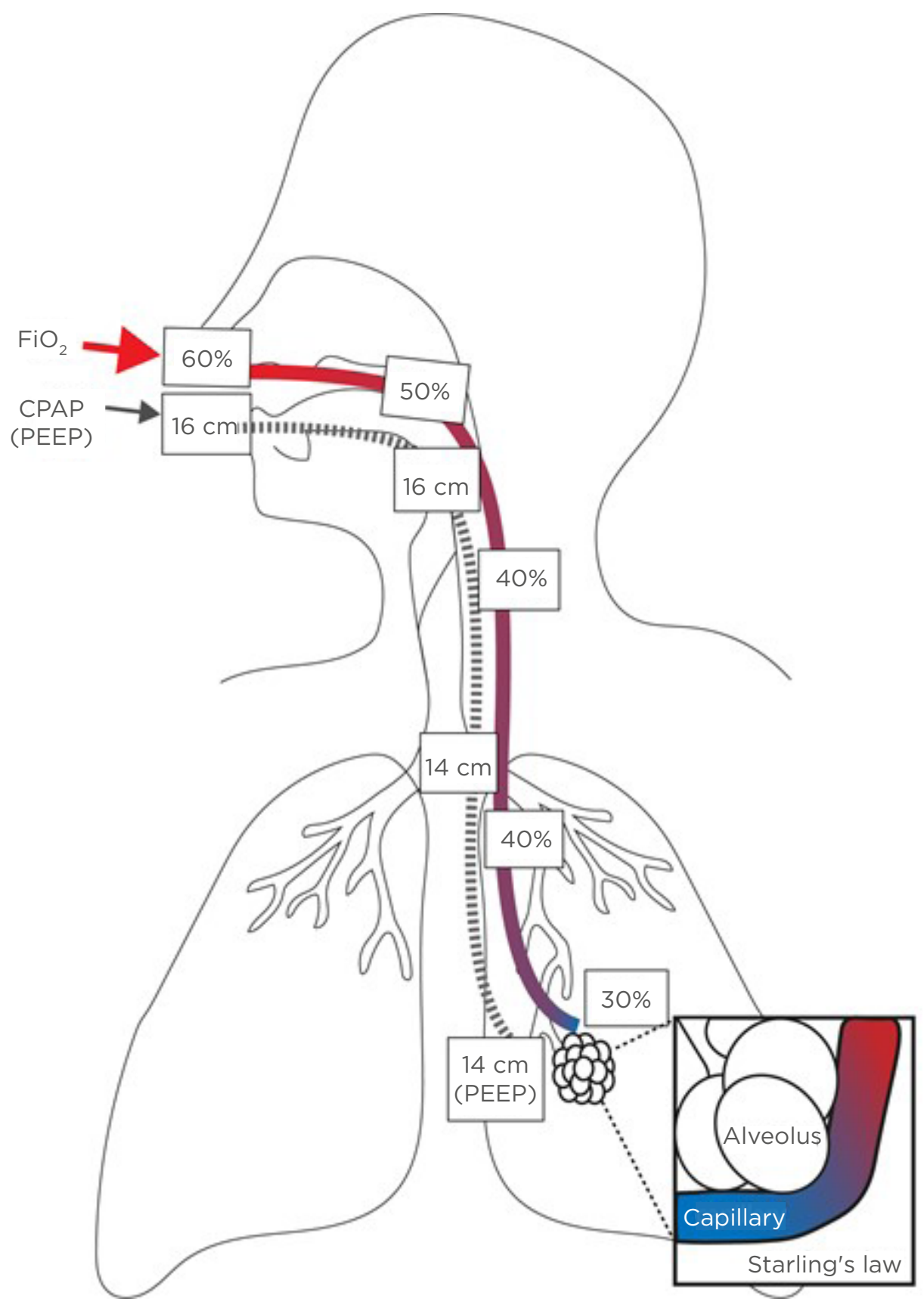

Figure 2: Combined benefits of airway pressure and oxygen.

CPAP: continuous positive airway pressure; $\mathrm{FiO}_{2}$ : fraction of inspired oxygen; PEEP: positive end-expiratory pressure.

A meta-analysis showed that systemic steroids improve mortality in critically ill patients with COVID-19, likely due to cytokine storms and because steroids reduce bystander immunerelated alveolar damage..$^{59}$

Pathogenic T cells and inflammatory monocytes incite cytokine storms with large amounts of IL-6; therefore, monoclonal antibodies that target the IL- 6 pathways may potentially curb these inflammatory storms. Tocilizumab, a monoclonal antibody against IL-6, appears to reduce mortality by approximately $24 \%$ and duration of organ support by around 1 week, in patients in intensive care units who need ventilatory support and have already received steroids (number needed to treat: 12). ${ }^{60}$ Tocilizumab may not prove to be so effective in patients less critically ill and who only require oxygen. ${ }^{61}$ 
Other inflammatory and immunomodulatory drugs blocking endothelial activation and injury ${ }^{62}$ or boosting pulmonary epithelial cell clearance of alveolar oedema (Figure 1) may also be useful. ${ }^{63}$ Other therapies such as the antiviral drugs remdesivir, lopinavir, hydroxychloroquine, and interferon have not been shown to reduce mortality or initiation of ventilation. ${ }^{64}$ Convalescent plasma and hyperimmune immunoglobulins are still being tested.
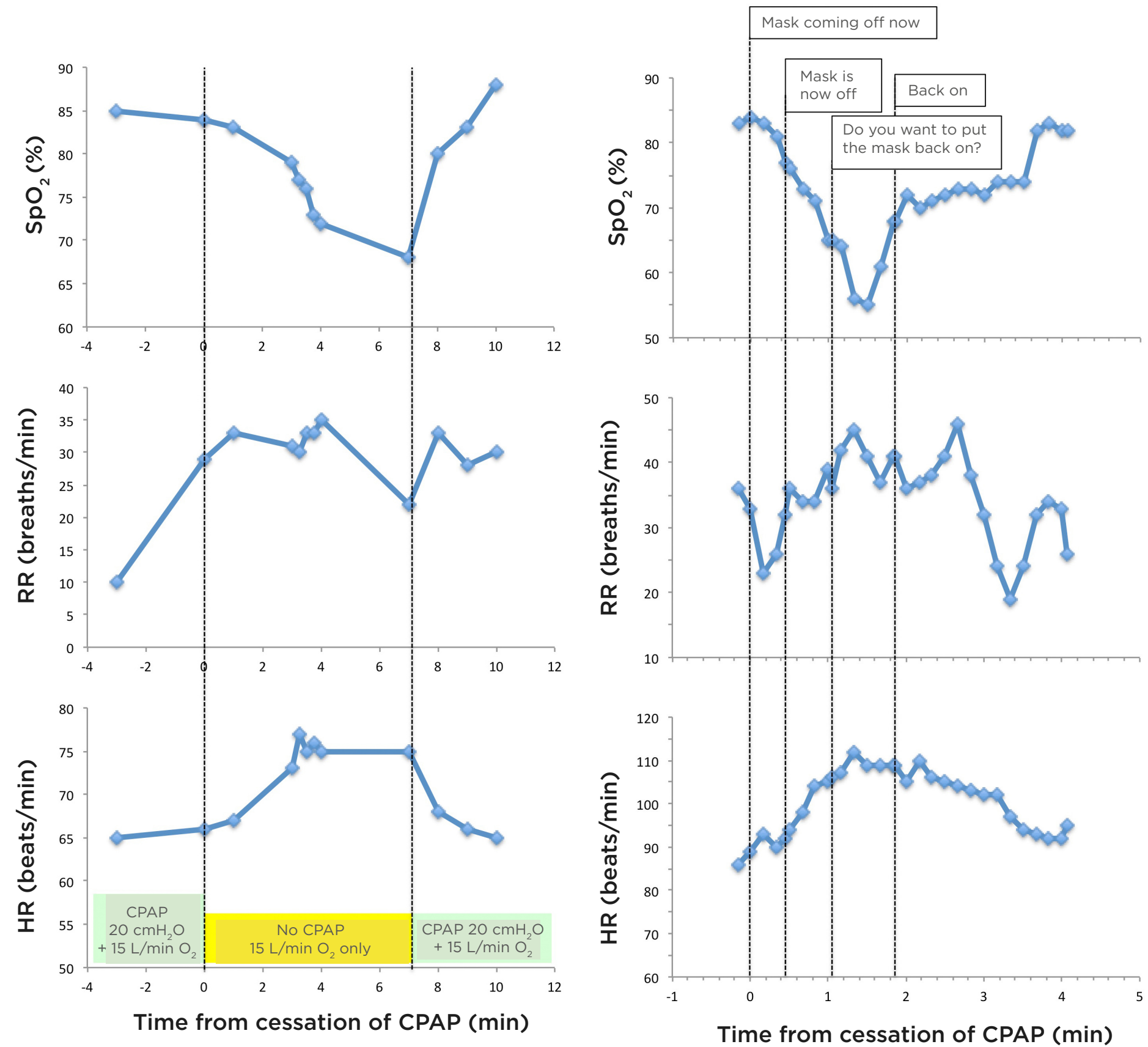

Figure 3: Changes in arterial oxygen saturation (skeletal muscle oxygen saturation, pulse oximetry), RR, and HR, on replacement of CPAP with high-flow oxygen during clinical care (to allow eating and drinking), and subsequent reintroduction of CPAP.

Comments on traces for Patient 3 were taken from audio recording coincident with cardiorespiratory monitoring. CPAP: continuous positive airway pressure; HR: heart rate; $\mathrm{RR}$ : respiratory rate; $\mathrm{SpO}_{2}$ : arterial oxygen saturation. 


\section{CLINICAL CASES}

The following clinical cases illustrate the pathophysiology and response to CPAP treatments.

A 59-year-old male was brought to hospital by ambulance. He was mildly overweight and had controlled hypertension. On arrival, his peripheral capillary oxygen saturation $\left(\mathrm{SpO}_{2}\right)$ was $67 \%$, despite receiving oxygen $15 \mathrm{~L} / \mathrm{min}$ via a nonrebreather mask. Arterial blood gases confirmed severe Type 1 respiratory failure (partial pressure of arterial oxygen: $5.3 \mathrm{kPa}$; partial pressure of $\mathrm{CO}_{2}: 4.3 \mathrm{kPa}$ ). His quick sepsisrelated organ failure score was 1 (blood pressure: 130/60 mmHg; respiratory rate: 38 breaths/min; Glasgow coma score: 15) but despite hypoxia he was able to speak on the phone. $\mathrm{SpO}_{2}$ improved to $80 \%$ and respiratory rate reduced to 24 breaths per minute with nasal CPAP $\left(12 \mathrm{cmH}_{2} \mathrm{O}\right.$ pressure) with entrained oxygen at $15 \mathrm{~L} / \mathrm{min}$. Improvement in temperature and oxygenation occurred 5 hours after receiving $500 \mathrm{mg}$ of intravenous methylprednisolone. CT confirmed bilateral extensive ground-glass shadowing and confluent infiltrates. A swab was positive for viral RNA of SARS-CoV-2. He was not intubated but continued CPAP with HFNO for 15 days with gradual weaning of both. He received six further doses of high-dose steroids (pre-RECOVERY results) and was discharged after 21 days.

Figure 3 displays transcripts from real-time videos of ward monitors from Patient 2 (53- yearold male, nonsmoker) and Patient 3 (58-year-old male, nonsmoker). Neither had any known lung or heart disease. Both patients were receiving 15
$\mathrm{L}$ /min of oxygen entrained through their CPAP machine (Trilogy Evo, Philips, Amsterdam, the Netherlands) set at $12-15 \mathrm{cmH}_{2} \mathrm{O}$ pressure.

The graphs demonstrate abrupt falls in oxygen saturations and rises in pulse and respiratory rate, because the CPAP mask was removed to allow the patients to drink and take short breaks. Both were immediately switched to oxygen nasal cannulas at $15-20 \mathrm{~L} / \mathrm{min}$. Neither felt unduly breathless on removing their masks; however, Patient 3 reported feeling tired. Their $\mathrm{SpO}_{2}$ and pulse improved within 20 seconds of reapplying the positive pressure, suggesting that combining CPAP with HFNO is beneficial in people where oxygen alone is not sufficiently effective.

\section{CONCLUSION}

By relating underlying changes in epithelia, subsequent endothelial infection and dysfunction, and the pathophysiology of alveolar leak, the different disease stages and why certain combinations of treatments are most effective can begin to be understood. More importantly, the rapid increase in knowledge, based initially on observational physiology and now interventions, supports the concept of sequential epithelial and endothelial failure and explains differences in individuals over time and the variable response to treatments. Most importantly, understanding why and how pathophysiology changes so quickly allows for tailored interventions. Appropriate selection of early CPAP and timely immunosuppression should further improve outcomes. Certainly, one (treatment) size does not fit all, and one size will not fit one person over time.

\section{References}

1. Alhazzani W et al. Surviving Sepsis Campaign: guidelines on the management of critically ill adults with coronavirus disease 2019 (COVID-19). Crit Care Med. 2020;48(6):e440-69.

2. World Health Organization (WHO). WHO coronavirus disease (COVID-19) dashboard. Available at: https:// covid19.who.int. Last accessed: 9 February 2021

3. Giwa AL et al. Novel 2019 coronavirus SARS-CoV-2 (COVID-19): an updated overview for emergency clinicians. Emerg Med Pract. 2020;22(5):1-28.

4. Bao $L$ et al. The pathogenicity of SARS-CoV-2 in hACE2 transgenic mice. Nature. 2020;583:1-20.

5. Hoffmann $M$ et al. SARS-CoV-2 cell entry depends on ACE2 and TMPRSS2 and is blocked by a clinically proven protease inhibitor. Cell. 2020;181:271-8.

6. Matthay MA et al. Lung epithelial fluid transport and the resolution of pulmonary edema. Physiol Rev.
2002;82:569-600.

7. Wu J et al. Chest CT findings in patients with coronavirus disease 2019 and its relationship with clinical features. Invest Radiol. 2020;55:25761.

8. Schaller T et al. Postmortem examination of patients with COVID-19. JAMA. 2020;323(24):251820

9. Shi $\mathrm{H}$ et al. Radiological findings from 81 patients with COVID-19 pneumonia in Wuhan, China: a descriptive study. 
Lancet Infect Dis. 2020;20:425-34.

10. Zhao W et al. Relation between chest $\mathrm{CT}$ findings and clinical conditions of coronavirus disease (COVID-19) pneumonia: a multicenter study. AJR Am J Roentgenol. 2020;214(5):1072-7.

11. $\mathrm{Xu} \mathrm{Z}$ et al. Pathological findings of COVID-19 associated with acute respiratory distress syndrome. Lancet Respir Med. 2020;8:420-2.

12. Peng $Q-Y$ et al. Findings of lung ultrasonography of novel corona virus pneumonia during the 2019-2020 epidemic. Intensive Care Med. 2020;46(5):849-50.

13. Pan $\mathrm{F}$ et al. Time course of lung changes at chest CT during recovery from coronavirus disease 2019 (COVID-19). Radiology. 2020;295:71521.

14. National Health Service (NHS) Health Education England. East of England School of Radiology. Available at: https://heeoe.hee.nhs.uk/radiology. Last accessed: 9 February 2021.

15. Hou YJ et al. SARS-CoV-2 reverse genetics reveals a variable infection gradient in the respiratory tract. Cell. 2020;182(2):429-46.e14.

16. Kanne JP et al. Essentials for radiologists on COVID-19: an updateradiology scientific expert panel. Radiology. 2020;296(2):E113-E114.

17. Buja LM et al. The emerging spectrum of cardiopulmonary pathology of the coronavirus disease 2019 (COVID-19): report of 3 autopsies from Houston, Texas, and review of autopsy findings from other United States cities. Cardiovasc Pathol. 2020;48:107233.

18. Menter T et al. Post-mortem examination of COVID19 patients reveals diffuse alveolar damage with severe capillary congestion and variegated findings of lungs and other organs suggesting vascular dysfunction. Histopathology. 2020;77(2):198-209.

19. Pernazza A et al. Early histologic findings of pulmonary SARSCoV-2 infection detected in a surgical specimen. Virchows Arch. 2020;477(5):743-8.

20. Tian S, Xiao S-Y. Pathology of 2019 novel coronavirus pneumonia: a dynamic disease process. J Thorac Oncol. 2020;15(5):e67-8.

21. Zhang $\mathrm{H}$ et al. Histopathologic changes and SARS-CoV-2 immunostaining in the lung of a patient with COVID-19. Ann Intern Med. 2020;172(9):629-32.

22. Lax SF et al. Pulmonary arterial thrombosis in COVID-19 with fatal outcome: results from a prospective, single-center, clinicopathologic case series. Ann Intern Med. 2020;173(5):350-61.

23. Thüsen von der J, van der Eerden M. Histopathology and genetic susceptibility in COVID-19 pneumonia. Eur J Clin Invest. 2020;50(7):e13259.

24. Tian $S$ et al. Pathological study of the 2019 novel coronavirus disease (COVID-19) through postmortem core biopsies. Mod Pathol. 2020;33(6):1007-14

25. Chen $\mathrm{N}$ et al. Epidemiological and clinical characteristics of 99 cases of 2019 novel coronavirus pneumonia in Wuhan, China: a descriptive study. Lancet. 2020;395:507-13.

26. Wang $D$ et al. Clinical characteristics of 138 hospitalized patients with 2019 novel coronavirus-infected pneumonia in Wuhan, China. JAMA. 2020;323:1061-9.

27. Guan W-J et al. Clinical characteristics of coronavirus disease 2019 in China. N Engl J Med. 2020;382:1708-20

28. Zhou $\mathrm{F}$ et al. Clinical course and risk factors for mortality of adult inpatients with COVID-19 in Wuhan, China: a retrospective cohort study. Lancet. 2020;395:1054-62.

29. Pons $S$ et al. The vascular endothelium: the cornerstone of organ dysfunction in severe SARS-CoV-2 infection. Crit Care. 2020;24:353-8.

30. Varga $Z$ et al. Endothelial cell infection and endotheliitis in COVID-19. Lancet. 2020;395:1417-8.

31. Ackermann $\mathrm{M}$ et al. Pulmonary vascular endothelialitis, thrombosis, and angiogenesis in COVID-19. N Engl J Med. 2020;383:120-8.

32. Kaur $\mathrm{S}$ et al. The enigma of endothelium in COVID-19. Front Physiol. 2020;11:989

33. ARDS Definition Task Force; Ranieri $\mathrm{VM}$ et al. Acute respiratory distress syndrome: the Berlin definition. JAMA. 2012;307(23):2526-33.

34. Liu $X$ et al. Ventilatory ratio in hypercapnic mechanically ventilated patients with COVID-19 associated ARDS. Am J Respir Crit Care Med. 2020;201(10):1297-9

35. McGuinness $G$ et al. High incidence of barotrauma in patients with COVID-19 infection on invasive mechanical ventilation. Radiology. 2020;297(2):E252-62

36. Roesthuis $L$ et al. Advanced respiratory monitoring in COVID-19 patients: use less PEEP! Crit Care. 2020;24:230-4

37. National Health Service (NHS). Clinical guide for the management of critical care for adults with COVID-19 during the coronavirus pandemic. Available at: https://www.nice.org uk/Media/Default/About/COVID-19/ Specialty-guides/Specialty-guide Adult-critical-care.pdf. Last accessed: 9 February 2021.

38. Grasselli $\mathrm{G}$ et al. Baseline characteristics and outcomes of 1591 patients infected with SARS-CoV-2 admitted to ICUs of the Lombardy region, Italy. JAMA. 2020;323:1574

39. Arentz $\mathrm{M}$ et al. Characteristics and outcomes of 21 critically ill patients with COVID-19 in Washington state. JAMA. 2020;323:1612-4.

40. Gattinoni L et al. Covid-19 does not lead to a "typical" acute respiratory distress syndrome. Am J Respir Crit Care Med. 2020;201(10):1299-300.

41. Pfeifer $M$ et al. [Position paper for the state of the art application of respiratory support in patients with COVID-19 - German Respiratory Society]. Pneumologie. 2020;74(6):337-57. (In German).

42. Miserocchi G. Lung interstitial pressure and structure in acute hypoxia. Adv Exp Med Biol. 2007;618:141-57.

43. Marini JJ, Gattinoni L. Management of COVID-19 respiratory distress. JAMA. 2020;323(22):2329-30.

44. Shekar K et al. Extracorporeal Life Support Organization Coronavirus Disease 2019 Interim Guidelines: a consensus document from an international group of interdisciplinary extracorporeal membrane oxygenation providers. ASAIO J. 2020;66(7):707-21.

45. Bennett $J$ et al. Respiratory advice for the non-respiratory physician in the time of COVID-19. Clin Med. 2020;20:251-5.

46. Paintal AS. Mechanism of stimulation of Type J pulmonary receptors. J Physiol. 1969;203:511-32.

47. Solaimanzadeh I. Acetazolamide, nifedipine and phosphodiesterase inhibitors: rationale for their utilization as adjunctive countermeasures in the treatment of coronavirus disease 2019 (COVID-19). Cureus. 2020;12:e7343.

48. Kirkman E, Watts S. Characterization of the response to primary blast injury. Philos Trans R Soc Lond B Biol Sci. 2011;366(1562):286-90.

49. Jugg BJA et al. The injured lung: clinical issues and experimental models. Philos Trans R Soc Lond B Biol Sci. 2011;366(1562):306-9.

50. Brochard L et al. Mechanical ventilation to minimize progression of lung injury in acute respiratory failure. Am J Respir Crit Care Med. 2017:195:438-42.

51. Richardson S et al. Presenting characteristics, comorbidities, and outcomes among 5700 patients hospitalized with COVID-19 in the New York City area. JAMA. 2020;323:2052-9.

52. Wagner PD, West JB. Effects of diffusion impairment on $\mathrm{O}_{2}$ and $\mathrm{CO}_{2}$ time courses in pulmonary capillaries. J Appl Physiol. 1972;33:62-71.

53. Arulkumaran $\mathrm{N}$ et al. Use of non- 
invasive ventilation for patients with COVID-19: a cause for concern? Lancet Respir Med. 2020;8(6):e45.

54. Nightingale $R$ et al. Is continuous positive airway pressure (CPAP) a new standard of care for Type 1 respiratory failure in COVID-19 patients? A retrospective observational study of a dedicated COVID-19 CPAP service. BMJ Open Respir Res. 2020;7(1):e000639.

55. Villarreal-Fernandez E et al. A plea for avoiding systematic intubation in severely hypoxemic patients with COVID-19-associated respiratory failure. Crit Care. 2020;24(1):337.

56. National Health Service (NHS). Guidance for the role and use of noninvasive respiratory support in adult patients with COVID-19 (confirmed or suspected). Available at: https://www. nice.org.uk/Media/Default/About/ COVID-19/Specialty-guides/specialty-
guide-NIV-respiratory-supportand-coronavirus.pdf. Last access: 9 February 2021

57. Graham S et al. Continuous positive airway pressure: an early intervention to prevent phosgene-induced acute lung injury. Toxicol Lett. 2018;293:120-

58. RECOVERY Collaborative Group. Dexamethasone in hospitalized patients with COVID-19 preliminary report. N Engl J Med. 2020:DOI:10.1056/NEJMoa2021436.

59. WHO Rapid Evidence Appraisal for COVID-19 Therapies (REACT) Working Group; Sterne JAC et al. Association between administration of systemic corticosteroids and mortality among critically ill patients with COVID-19: a meta-analysis. JAMA. 2020;324:1330-41.

60. The REMAP-CAP Investigators; Gordon AC et al. Interleukin-6 receptor antagonists in critically ill patients with COVID-19 - preliminary report. medRxiv. 2021;DOI:10.1101/202 1.01.07.21249390. [Preprint].

61. Stone $\mathrm{JH}$ et al. Efficacy of tocilizumab in patients hospitalized with COVID-19. N Engl J Med. 2020;383:2333-44.

62. Simonsen $U$ et al. Emerging roles of calcium-activated $\mathrm{K}$ channels and TRPV 4 channels in lung oedema and pulmonary circulatory collapse. Acta Physiol. 2017;219:176-87.

63. He J et al. Rosiglitazone promotes ENaC-mediated alveolar fluid clearance in acute lung injury through the PPARY/SGK1 signaling pathway. Cell Mol Biol Lett. 2019;24:35.

64. WHO Solidarity Trial Consortium Pan $\mathrm{H}$ et al. Repurposed antiviral drugs for COVID-19 - interim WHO solidarity trial results. N Engl J Med. 2020;NEJMoa2O23184. 